\title{
Aportes del concepto de educación en Luhmann y su vinculación con el estudio de la exclusión
}

\author{
Contributions of the Concept of Education in Luhmann and its Relationship with the \\ Study of Exclusion
Contribuições do conceito de educação em Luhmann e sua vinculação com o estudo da exclusão

\author{
Julio Labraña
}

Universidad Federico Santa María, Hualpén, Chile. Telf.: 965307526. Correo electrónico:

Labrana.J07@gmail.com

\section{RESUMEN}

La sociología de la educación es una de las especialidades más antiguas de la teoría sociológica. Derivado de las anotaciones de los clásicos, existe hoy un consenso entre teorías funcionalistas, críticas y de capital humano en considerar la educación como central en la mantención de la desigualdad en la sociedad. El presente artículo da cuenta de la descripción de la educación desde la teoría de sistemas sociales como una alternativa epistemológica, haciendo notar las posibilidades que ofrece la diferenciación entre codificación y programación para el estudio de la exclusión.

Palabras clave: educación, sistema, Luhmann, exclusión.

\begin{abstract}
The sociology of education is one of the oldest specialties of sociological theory. Derived from the annotations of the classics, there is a consensus between functionalist theories, critical theories and human capital theories in order to consider education as central to the maintenance of inequality in society. This article reports a description of the education from the theory of social systems as a epistemological alternative, noting the potential of making an analytical difference between coding and programming for the study of exclusion..
\end{abstract}

Key words: education, system, Luhmann, exclusion.

\section{RESUMO}

A sociologia da educação é uma das especialidades mais antigas da teoria sociológica. Derivado das anotações de clássicos, há um consenso entre teorias funcionalistas, críticas e de capital humano em considerar a educação como eixo central para a manutenção da desigualdade social. Consegue-se a descrição da educação desde a teoria de sistema sociais como alternativa epistemológica, evidenciando as possibilidades que oferece a diferenciação entre codificação e programação para o estudo da exclusão.

Palavras chave: educação, sistema sociais, Luhmann, exclusão. 


\section{INTRODUCCIÓN}

El propósito de las siguientes reflexiones es exponer parte del panorama general de la sociología de la educación para relacionar las interrogantes que plantea con los conceptos ofrecidos por la teoría de sistemas del sociólogo alemán Niklas Luhmann y despejar parte de los prejuicios asociados a su utilización. La intención del artículo es modesta: no es el objetivo realizar un recuento exhaustivo de las críticas, como tampoco hacer una síntesis de los estudios empíricos sobre el tema desde la teoría de sistemas como ha sido realizado en otros trabajos (Massé, 2007; Qvortrup, 2005). Más bien, estas serán tomadas en cuenta únicamente como medios para ajustar la observación de la desigualdad en la educación y de esta forma incorporar irritaciones que puedan hacer necesaria la formulación de correcciones en la teoría de sistemas.

Para abordar este objetivo, el artículo se inicia con "La unidad del debate por la educación", donde doy cuenta de las discusiones tradicionales en la sociología de la educación y los presupuestos compartidos que presentan perspectivas teóricas tecnocráticas, críticas y funcionalistas en su concepción del aparato educativo como espacio privilegiado de la sociedad (I). A continuación, expondré las diferencias de este paradigma respecto a la educación en la teoría de sistemas en el marco de la diferenciación funcional bajo el título "El sistema de la educación como un sistema social" (II). Posteriormente, en "La utilización de la forma inclusión/exclusión en el sistema de la educación", (III) compararé cuatro formulaciones teóricas orientadas a comprender el problema de la desigualdad en la educación (Ossandon, Robles, Mascareño, Michailakis y Reich) para finalmente concluir con una breve reflexión sobre lo expuesto (IV).

\section{LA UNIDAD DEL DEBATE POR LA EDUCACIÓN}

La preocupación por la educación tiene larga data en la sociología. Ya en los escritos de los primeros teóricos sociales como Saint-Simon y Comte, queda de manifiesto un planteamiento epistemológico sobre las funciones que les correspondía cumplir a la educación en la sociedad contemporánea. Esta observación tiene sus orígenes en los cambios sociales de la época, particularmente la ruptura que significó la transición desde la sociedad aristocrática del Antiguo Régimen a la sociedad industrial como pérdida de los fundamentos sociales naturales. En las sociedades estratificadas la naturalidad de la posición social estaba fundamentada en la inmovilidad entre estratos que reglamentaba en forma clara las posibilidades de comunicación individuales (Luhmann, 1998). Aún en el caso de que este ordenamiento fuese percibido como problemático mediante tumultos, siempre era posible una última apelación a la Iglesia Católica como garante de la presencia de un mínimo de valores y normas de convivencia.

Derivado de lo anterior, la sociología de la educación desde sus inicios se fue constituyendo como una sociología de las instituciones escolares marcando con un especial énfasis funciones orientadas al control social y la instauración moral. El primer libro clásico sobre el tema, "Educación y sociología" de Durkheim, rechazaba las idealizaciones en boga de Kant sobre la educación para destacar, en cambio, la necesidad de un escuela que educase para las necesidades de diferenciación producto de la división del trabajo e imposición de una unidad moral que suponía la ausencia de integración. Debido a sus 
características de obligatoriedad e intencionalidad específicas, la educación se observó como el espacio donde frenar las tendencias anómicas mediante la homogeneización de una serie de estados físicos, intelectuales y morales en la población. Según el mismo autor, la función de la educación estaba en "desarrollar en el alumno la aptitud general pará la moralidad, las disposiciones fundamentales que están en la raíz de la vida moral y constituir en el alumno el agente moral, dispuesto a las iniciativas que son condición general del progreso" (Durkheim, 1991).

A partir de las transformaciones industriales ocurridas durante el siglo XX, las teorías funcionalistas profundizaron sus planteamientos en la función de la escuela como garante de unidad moral y diferenciación laboral. Con especial énfasis la educación, adquirió una mayor importancia tras la segunda guerra mundial, al trasladarse la competencia entre potencias por la hegemonía militar desde el ámbito económico al de la producción tecnológica (Bonal, 1998: 30). En este contexto, la reflexión de Parsons propone un mayor desarrollo teórico sobre las funciones de la educación. Para el sociólogo norteamericano el problema que la educación debe satisfacer en la sociedad moderna es la necesidad de internalizar compromisos normativos, a la vez que promover la asignación de récursos para la estructura de roles o, como lo señala él mismo, "consérvar los valores más fundamentales de la sociedad" y promover el "desempeño de un tipo específico de rol dentro de la estructura social" (Parsons, 1976).

La forma mediante la cual estos objetivos debían lograrse era a partir de una evaluación centrada únicamente en logros académicos derivados de la capacidad propia de cada estudiante y no sobre factores como el sexo o el estatus socioeconómico. Por lo tanto, el sistema educativo moderno, único con estas características, sería el único que pudiese realizar las funciones centrales en el ordenamiento de la sociedad. En primer lugar, hace posible la emancipación del niño de la influencia restringida de la familia mediante la asignación en los primeros años de un profesor único, largas jornadas escolares y relación con pares no familiares. Esto promueve el remplazo de la estructura social de la familia orientada según criterios particularistas por la orientación universalista del profesor, asegurando las expectativas normativas mínimas que la convivencia en la sociedad requeriría. Por otra parte, el tiempo de permanencia en la escuela suscita con mayor probabilidad la internalización de determinadas normas, materias y costumbres. En último lugar, puesto que la escuela únicamente diferencia según el desempeño expresado en los resultados de evaluaciones y exámenes aplicados por un profesor representante del mundo adulto sobre los alumnos, la diferenciación social toma una apariencia que permite mitigar las tensiones que el mismo genera (Parsons, 1976). A partir de estas características, la perspectiva de Parsons asume la centralidad del aparato educativo en la regulación de los problemas provocados por la diferenciación de la sociedad.

Particularmente desde la década de los 70', la neutralidad de las políticas educativas fue puesta en cuestión por un serie de movimientos sociales que, retomando la teoría marxista, criticaban el fracaso de la educación como mecanismo de distribución e imposición de solidaridad en la sociedad. Esta situación condujo a los gobiernos europeos y estadounidense a investigar el fenómeno, buscando encontrar la causa en la falta de recursos económicos destinados a la educación. Sin embargo, contrario a estos supuestos, el Informe Coleman (1966) indicó que la explicación de la correspondencia entre clase social y resultados académicos estaba en la privación cultural de las familias y no en el tipo de escuelas al que asistían. Tanto este como otros estudios que continuaron en esta 
tesis resultaron notablemente problemáticos para las premisas de las teorías funcionalistas. La investigación de Jencks (1972), Inequality, señaló la desconexión existente entre posición educativa (desempeño), estatus ocupacional e ingresos, para concluir la incapacidad de los resultados académicos de describir la forma de inserción en el mundo laboral. En idéntica dirección, Thurow (1983) hacía notar que, pese a haber aumentado el nivel educativo de la población entre 1950 y 1970 en los países del tercer mundo, la pobreza no había dejado de incrementarse. De esta forma, quedaba en entredicho la función de diferenciación laboral derivada del mérito académico.

Sin embargo, pese a poner en cuestión los supuestos funcionalistas de idéntica accesibildad a los contenidos educativos, la diferenciación producto de criterios académicos y la conexión entre resultados escolares y posición en la estructura laboral, los estudios anteriores mantuvieron intacta la concepción de un sistema educativo que, pese a ser imperfecto, era neutral en su imposición de unidad. Las teorías críticas, en cambio, se presentaron a sí mismas como desveladoras de la función de la educación para mantener las diferencias entre clases sociales. La desigualdad no era una consecuencia irregular del funcionamiento del aparato escolar, sino, más bien, del rol correspondiente de la educación en determinada etapa de desarrollo del capitalismo.

La idea central de los textos de Bourdieu sobre la educación es que la arbitrariedad en la selección de determinadas formas de cultura favorece sistemáticamente a los individuos provenientes de familias con un mayor capital cultural y segrega a aquellos de menores recursos que carecen de este. En 1964 el autor publica junto a Passeron la investigación "Los estudiantes y la cultura", donde señala que las principales limitaciones para los estudiantes de bajos ingresos de la Universidad de Paris no son de tipo económico, como podría argumentar el funcionalismo, sino culturales, pues las evaluaciones presuponen capacidades cuya adquisición en familias de bajo capital cultural es menos probable (Bourdieu y Passeron, 2003: 45). En efecto, en los exámenes los profesores universitarios tenderían a valorar la creatividad y la asociación abstracta por sobre la aplicación empírica de conceptos. Dado que los alumnos provenientes de familias de menores recursos han sido socializados para orientarse a aspectos prácticos y no intelectuales, esta desventaja social se realiza como un fracaso del propio alumno, en tanto el desempeño superior de alumnos provenientes de familias de mejores ingresos no se observa como consecuencia del contexto familiar, sino de dones y talentos individuales.

La selección de estos criterios de evaluación no se deriva de una jerarquia del conocimiento, sino que es el producto de luchas de poder en la estructura social que, por tanto, terminan otorgando legitimación a una clase por sobre otra. Como señala el sociólogo francés, "por el hecho de que corresponden a los intereses materiales y simbólicos de grupos o clases distintamente situados en las relaciones de fuerza, estas acciones pedagógicas tienden siempre a reproducir la estructura de la distribución del capital humano entre esos grupos o clases, contribuyendo con ello a la reproducción de la estructura social" (Bourdieu, 1977). De esta forma, la supuesta autonomía de la educación respecto a la sociedad es sólo el modo mediante el cual esta aseguraría la objetividad de sus intenciones, al ocultar el hecho de que es en la escuela y la universidad donde la diferencia económica entre grupos sociales adquiere una forma menos problemática, al asociarse al capital cultural centralizando el logro académico como criterio de diferenciación entre los alumnos. 
Donde Bourdieu establece la arbitrariedad de los criterios educativos, Bernstein se interroga sobre los mecanismos específicos que favorecen y desfavorecen a ciertas clases sociales. Debido a que la posición de las familias en la división de trabajo determina el lenguaje que estas utilizan, la institucionalización en el aparato educativo de cierto código lingüístico termina por posibilitar determinadas formas de control social. Para el sociólogo británico existen dos formas lingüísticas opuestas: el código restringido y el código elaborado o formal. Mientras el primero es utilizado preferentemente por la clase baja, primando expresiones gramaticales cortas, fuertemente dependientes de las experiencias vitales, el segundo está arraigado en las clases medias y altas, siendo preferenciales las construcciones gramaticales complejas, la utilización de pronombres impersonales y el desarrollo de una mayor conceptualización (Bernstein, 1985: 45).

Dado que el sistema educativo ha evolucionado con pretensiones universalistas y de abstracción, en el código elaborado rige el currículo, la pedagogía y las formas de evaluación, dejando en desventaja a quienes se hubiesen socializado en un código lingüístico más restringido. Por otra parte, debido a la centralidad de la educación en la sociedad actual, las instituciones escolares y su selección del código elaborado promueven la mantención del orden social, pues, como menciona Bernstein (1958), "cómo una sociedad selecciona, distribuye, transmite y evalúa el conocimiento educativo que ella considera debe ser público, refleja tanto la distribución del poder como los principios del control social".

Desde una perspectiva distinta, pero conservando el análisis crítico de la educación, Althusser señala las funciones de la educación en la sociedad capitalista a partir de la introducción del concepto de aparatos ideológicos del Estado. Junto con la iglesia y la familia, la escuela se presentaría como el espacio ideal en el que imponer la ideología burguesa en el conjunto de la sociedad. Dado que está menos subordinada a las relaciones con el Estado, la educación parece ser "relativamente autónoma", ofreciendo así un campo para la expresión de contradicciones que hagan evidentes los efectos de los choques entre la lucha de clases capitalista y la lucha de clase proletaria. Sin embargo, no por esto debe entenderse que la educación es un aspecto secundario en la mantención de las relaciones de poder. De hecho, precisamente por el rol central que cumple en la actualización ideológica de las conciencias, la transformación de estas sin mediar cambios en la estructura de los medios de producción es imposible (Althusser, 1985: 308).

La tesis de Bowles y Gintis se basa en la correspondencia existente entre la estructura económica y la superestructura ideológica en el ámbito de la educación. Los autores señalan la presencia de un continuo entre las relaciones escolares y las de producción, sea en torno a las jerarquías, como frente a los grados de injerencia, control o productividad. De esta forma, la relación entre profesor y alumno actuaría como una metáfora de la relación entre el dueño de los medios de producción y el trabajador, así como la participación del alumnado en los planes de estudio sería tan vacía como la del trabajador en los planes futuros de desarrollo en la empresa (Bowles y Gintis, 1976: 175).

Esto les permite a los autores asignarle al sistema educativo la función de constituir las formas de personalidad necesarias para la estructura productiva capitalista. Los individuos de clases inferiores aprenden en la escuela a obedecer normas pasivamente, en tanto que los hijos las familias de clases de mayores recursos desarrollan una mayor autonomía para emprender. Como señalan los autores, "las relaciones jerárquicas están reflejadas en las línea de autoridad verticales que van de administradores a maestros y de maestros a estudiantes. El trabajo enajenado se refleja en la falta de control que tiene 
el estudiante sobre la educación, la enajenación de éste sobre el contenido de sus planes de estudio y la motivación escolar a través del sistema de recompensas externas, en lugar de mediante la integración del estudiante" (Bowles y Gintis, 1976). En consecuencia, la escuela es comprendida como el espacio donde la socialización del individuo hacia las normas requeridas por la estructura de clases se realiza.

Conviven junto a las propuestas funcionalista y crítica una visión tecnocrática crecientemente dominante desde las reformas neoliberales, marcada por la recolección de datos económicos y promoción de políticas públicas, cuyo máximo exponente se encuentra en la teoría del capital humano. Los supuestos de esta teoría obedecen a una actualización del ideario funcionalista tras las variaciones en la estructura productiva al sector servicios, incorporando además una concepción de la educación como una inversión con tasas específicas de rentabilidad a nivel social (Bonal, 1998: 58).

Desde esta vertiente teórica, las investigaciones han partido de la observación de los aparatos educativos como el espacio ideal para intervenir y asegurar el progreso. Por ello fueron orientados por una serie de organismos internacionales, como la OCDE, UNESCO y el Banco Mundial (Bonal, 1998). En esta dirección podemos leer las transformaciones impuestas en la dictadura militar en nuestro país. En 1979, la Directiva de Educación señalaba que el Estado centraría sus esfuerzos en la educación básica, para así capacitar "buenos trabajadores, buenos ciudadanos y buenos patriotas". Tras el retorno a la democracia, la Comisión Brunner no abandonó las teorías del capital humano, apuntando esta vez a la educación con funciones de hacer posible la "vida civilizada de comunidad", así como el logro de la democracia y la erradicación de la pobreza, al hacer utilizables los talentos en provecho del país. En el mismo sentido, el economista Harald Beyer señala que los beneficios de una buena educación se encuentran en "contribuir a la competitividad y al crecimiento económico (...) elevando la productividad de nuestra fuerza de trabajo y potenciando el desarrollo de áreas de mayor valor agregado". Una buena educación también construye una "sociedad de mejores personas y puede ayudar a reducir la desigualdad económica que afecta a nuestro país" (Beyes, s/f). De esta forma, según los seguidores de las teorías del capital humano, la educación es el mejor espacio para desarrollar lo social fomentando la moral, el patriotismo, el crecimiento económico y las relaciones democráticas entre sus habitantes.

Puede este parecer un panorama sumamente rico para la reflexión sociológica, en tanto posibilita la convivencia de tres vertientes aparentemente opuestas entre sí. Mientras las perspectivas funcionalistas privilegian la observación de la unidad moral y diferenciación laboral que subyacen a los objetivos de la enseñanza, la visión crítica destaca cómo mediante estos mismos mecanismos se asegura la reproducción de una sociedad estratificada según clases sociales. La tercera opción tecnocrática decantaría por oposición en el desarrollo de políticas públicas orientadas al bienestar general de la sociedad mediante una mayor cantidad y calidad de organizaciones educativas.

Sin embargo, y esta es mi tesis, en todas las aproximaciones es posible encontrar un núcleo epistemológico común de la educación como el aspecto central de la sociedad donde es posible afianzar rígidamente una forma específica de relaciones sociales. En efecto, en las variantes funcionalistas, en ausencia de la socialización promovida por la escuela, la mantención de las pautas normativas básicas de convivencia es imposible. En el caso de las vertientes críticas, la educación cumple una función ideológica, en tanto asegura la mantención de la diferencia entre clases sociales y legitima el orden 
estratificado. Finalmente, en las concepciones derivadas del capital humano, una mejora en las condiciones de educación hace posible el logro estricto de una serie de beneficios en otros ámbitos de la sociedad, sean estos morales, políticos o económicos. De esta forma, en todas las teorías mencionadas la educación dirige al conjunto de la sociedad a un futuro mejor (teorías del capital humano y funcionalista) o peor (teorías críticas).

Considero posible proponer una interpretación diferente a esta unidad teórica mediante un enfoque que rescate las diferencias de la educación y su autonomía operativa respecto a otros ámbitos de la sociedad. Particularmente, me oriento en este objetivo según la teoría de sistemas sociales del sociólogo alemán Niklas Luhmann, especialmente a partir de la diferenciación funcional como característica principal de la sociedad contemporánea.

\section{EL SISTEMA DE LA EDUCACIÓN COMO UN SISTEMA SOCIAL}

La teoría de sistemas sociales propuesta por Niklas Luhmann se basa en la comprensión de la sociedad como el total de comunicaciones que posibilitan la emergencia de sistemas operativamente cerrados, autorreferenciales y autopoiéticos, encargados del cumplimiento de una función específica. En la sociedad contemporánea uno de estos sistemas es la educación. Comenzaré esta sección con una descripción de la teoría de la diferenciación sistémica, para después dar cuenta de las especificidades de este sistema funcional.

La diferenciación de la sociedad es la forma mediante la cual la propia sociedad resuelve sus problemas auto creados mediante la reintroducción de la diferencia entre sistema y entorno en el interior del sistema (Luhmann, 1998: 78). Luhmann distingue tres formas de combinación de la asimetría entre sistema y entorno respecto a la diferencia entre igualdad y desigualdad: segmentación, estratificación y diferenciación funcional. Dado que la preocupación del presente trabajo es abordar la sociedad contemporánea, me concentraré únicamente en el último desarrollo evolutivo. En esta la sociedad se organiza en subsistemas autónomos, clausurados operativamente y con un medio de operaciones único, asociado a su función específica. Este es el caso de la verdad en la ciencia, el amor en las relaciones de intimidad, el dinero en la economía o el poder en la política. La autopoiesis del sistema queda determinada, por tanto, en que cada sistema crea los elementos que le permiten mantener su unidad frente al entorno. Dado que toda función requiere ser cumplida con idéntica premisa para la interdependencia de los subsistemas, la sociedad no puede conceder primacía a ninguna de ellas, resultando por tanto una sociedad carente de centro único, esto es, policéntrica.

Estas consideraciones nos alejan ya de las observaciones de la educación como guía y centro. Por lo demás, no es posible en una sociedad orientada según la crítica como la actual argumentar que la unidad sistémica se realiza en la representación (representatio identitatis) de un subsistema por sobre la totalidad o, para decirlo más laxamente, de una parte respecto al todo (Luhmann y Schorr, 1996). En este sentido, en una sociedad funcionalmente diferenciada los sistemas sociales no pueden apelar a coordinar sus operaciones según los criterios de otros sistemas. Los sistemas funcionales son desiguales entre sí (la política no puede encargarse de la economía), si bien presuponen la igualdad en torno a la inclusión universal en el acceso a las funciones de cada sistema para discriminar según la función. En efecto, la tendencia es a estructurar la desigualdad únicamente como producto 
de las operaciones en el interior de cada sistema, con lo que la estratificación tiende a perder primacía como criterio de diferenciación. De esta forma, el lado reverso de esta característica de la diferenciación funcional es que los sistemas se vuelven negligentes respecto a variaciones que no correspondan al medio de operación del sistema. En las operaciones económicas no son relevantes ni la moral ni las orientaciones políticas, asî como tampoco el sufrimiento personal (Luhmann, 1994).

Sin embargo, ¿cómo surgen los sistemas funcionales? La respuesta se encuentra en la emergencia de los medios simbólicamente generalizados, estructuras que permiten aumentar las posibilidades de aceptación de selecciones contingentes mediante la unión de motivación individual, con la selección social haciendo emerger un mundo estrecho de entendimientos comunes, expectativas complementarias y temas determinables (Luhmann, 1998: 107). En consecuencia, la sedimentación de expectativas asociadas a cada uno de los sistemas funcionales posibilita que la aceptación de la comunicación sea mayor a lo que sería sin medios. Esto no implica que los medios generalizados limiten la selección a partir de la interiorización de 'pautas control' (Parsons), ni tampoco que se ubiquen en la función de coordinación del lenguaje (Mascareño, 2009: 182). Más bien, los medios permiten referir a la vez a procesos de atribución entre alter y ego y de institucionalización sistémica, promoviendo la actualización de determinadas cadenas de selección sin asegurarlas. En el caso de que las expectativas sean negadas, por ejemplo, respondiendo a una interacción económica con una declaración de amor, la cadena simplemente se disuelve o varía hacia una nueva estabilización.

Las bases mínimas en que se realiza la participación de los individuos en el mundo es mediante dos modalidades de atribución: vivencia y acción. Atribución, pues son producto de observaciones y no realidades plenas, en tanto esto omitiría la clausura operativa de los sistemas psíquicos. Por vivencia se entiende la atribución de la selectividad al entorno del sistema o la observación del pensamiento propio como vivencia interna, en tanto la acción consiste en atribuir la selección a una unidad externa u observar la corporalidad bajo la forma de la acción. Según estas formas de atribución, y si las selecciones de alter son consideradas como vivencia o acción, y si ego coordina con la selección de alter su propio actuar o vivencia, surgen las siguientes constelaciones sociales y su correspondiente medio simbólicamente generalizado:

Tabla 1. Constelaciones sociales surgidas a partir de Vivencias de Ego y Acción de Ego

\begin{tabular}{|c|c|c|}
\hline & Vivencia de Ego & Acción de Ego \\
\hline \multirow{2}{*}{ Vivencia de Alter } & Av Ev & Av Ea \\
\cline { 2 - 3 } & verdad; valores & amor; influencia \\
\hline \multirow{2}{*}{ Acción de Alter } & Aa Ev & Aa Ea \\
\cline { 2 - 3 } & propiedad/dinero; arte & poder/derecho \\
\hline
\end{tabular}

En el primer recuadro aparece la selección de vivencia de alter aceptada como motivación para la vivencia de ego dando lugar a los medios de la verdad y los valores. A continuación se muestra la coordinación entre la vivencia de alter y la acción de ego 
dando lugar al medio del amor. Esto implica que las acciones de ego deben confirmar las vivencias de alter (Mascareño, 2006). En tercer lugar está la acción de alter tomada como premisa para una vivencia de ego mediante la propiedad, el dinero y el arte. En último lugar se ve una acción de alter como antecedente de una acción de ego. Los medios correspondientes a esta constelación son el poder y derecho. Debido a que la evolución de la sociedad es un proceso constante, esta no puede ser considerada una lista acabada. Existen varios sistemas diferenciados o en proceso de diferenciación que no presentan un medio simbólicamente generalizado claro, como es el caso de la religión, el deporte, la solidaridad y los medios de comunicación de masas. En estas páginas nos interesa uno de los sistemas que carecen de medio en particular: la educación.

Cabe destacar que no es casual la ausencia de un medio simbólico asociado a la educación. Puesto que esta se define por la búsqueda de cambios en el entorno de la sociedad (sistema psíquico), no puede construir procesos comunicacionales de atribución de vivencias y acciones ${ }^{1}$. Por lo demás, el horizonte de evaluación es demasiado complejo y el grado de individualización que hay que conceder en la sociedad moderna es demasiado alto (Luhmann y Schorr, 1996: 146). Esto no impide, sin embargo, el desarrollo de mecanismos que permitan al sistema educacional incrementar la probabilidad de enlazamiento de comunicaciones mediante una mayor diferenciación frente a la sociedad. La tesis que sostengo en este apartado es que, pese a ciertas carencias respecto a otros sistemas funcionales, la educación, lejos de educar para la producción o reproducción total de la sociedad, lleva a cabo sus operaciones únicamente mediante construcciones propias. Más específicamente, la educación es un sistema que genera sus propias diferencias sin tener que importarlas del entorno o, lo que es lo mismo, sin plantearse como un continuo lineal respecto a la sociedad.

El sistema de la educación en la sociedad moderna lleva a cabo dos funciones aparentemente contradictorias: educación orientada al desarrollo de competencias y la observación de estas diferencias mediante la selección (Luhmann y Schorr, 2000). Mientras la comunicación educativa opera según el código de plausibilidad posible de enseñar/ imposible de enseñar, la selección lo hace mediante el código binario mejor/peor, que si bien puede variar en su programación, siempre distingue entre resultados aceptables y no aceptables. El acoplamiento de ambos códigos permite medir selectivamente, de acuerdo a prerrogativas formuladas en el sistema educativo, si el aprendizaje se logró o no, independiente de su correlato mental. De esta forma, respecto a un alumno que obtiene una nota inferior a 4.0 se presupone que no logró aprender lo que debía, independiente si esto se corresponde con las operaciones de su sistema psíquico.

Dado el grado de diferenciación del sistema de la educación, la asignación de diferencias de rendimiento no puede articularse según valores trascendentales como los ideales de perfección o moralización, pues la laxitud del medio de la moral imposibilita que esta pueda coordinar la evolución de la sociedad. En la diferenciación funcional no existen posiciones privilegiadas, no hay cumbre, centro coordinador ni un mundo de "perfecciones absolutas, axiomáticas e inmutables" (Luhmann y Schorr, 1990: 56). Más bien, lo

1 Sobre el medio de la educación existen propuestas de considerar la comunicación educativa en paralelo al medio simbólico del amor, es decir, como coordinación entre la vivencia del alumno y las acciones del profesor. De esta forma, las acciones del docente requerirían verse confirmadas en las variaciones psíquicas observadas en el alumno, catalizando así motivaciones de motivaciones. Véase Fernando Robles (2010: 37-40). 
que define a la autopoiesis sistémica es la diferencia entre codificación y programación. El código binario es un mecanismo que hace de la comunicación algo contingente (que puede ser así pero también de otro modo) que en la educación se realiza en la selección social. Unos alumnos pasan de curso y otros no; unos son elogiados y otros castigados y esta diferenciación permite atribuir posiciones en el sistema educativo.

Como ocurre también en otros sistemas funcionales, el código es negligente frente a comunicaciones que no se correspondan con su medio. Por ello, la posesión del dinero no asegura sabiduría ni cumplir con los requisitos necesarios para comenzar una carrera política, pese a que, debido a los resabios de estratificación, todavía puede suceder (Mascareño, 2005). Lo mismo sucede con el código de selección. La sociedad funcionalmente diferenciada, contrario a la estratificada, aspira a ser una e idéntica para todos -razón por la cual la influencia de desigualdades económicas resulta chocante. Ante la ausencia de la delimitación por el origen como destino de los individuos, se presupone en el funcionamiento del código un comienzo idéntico para todos los alumnos, una homogeneización del comienzo de forma tal, que las variaciones de los resultados entre estos sean únicamente producto de la educación.

Esto no implica la ausencia de un entorno siempre problemático. De hecho, la homogeneización del comienzo es antes un preriquisito del funcionamiento de los sistemas sociales antes que una característica de la sociedad. Sea por razones familiares o de orientación al aprendizaje, no todos los alumnos comienzan con una disposición favorable a la educación. Por lo demás, mediante la política o el derecho, no pocas veces se intervienen los parámetros del sistema. Sin embargo, la forma en la cual estas diferencias son incorporadas obedece a criterios educativos, esto es, de delimitación respecto a qué se incorporara del tiempo anterior al comienzo y qué se incorporara del tiempo posterior como pasado o futuro relevantes (Luhmann y Schorr, 1996: 54). Esta introducción de valores excluidos por el carácter binario del código se realiza en los programas. La pobreza, por ejemplo, no forma parte ni de los valores "mejor" o "peor", pero puede tematizarse en la educación mediante la creación de disposiciones de discriminación positiva, como el Plan de Escuelas Críticas y el Programa de 900 escuelas. Lo mismo sucede con las escuelas especiales para alumnos con capacidades especiales (Michailakis y Reich, 2009). De esta forma, mediante una conducta selectiva, los programas permiten actualizar los elementos del entorno que resulten relevantes para el sistema, siempre subordinándose a los valores y criterios del código.

Lamentablemente para la pedagogía, la separación entre codificación y programación implica la trivialización del alumno. Para comprender este procedimiento debemos aclarar la diferencia entre máquinas triviales y no triviales. Basta con saber que las primeras son las que se definen por responder en forma constante a un estímulo determinado, característica que asegura su control y predictibilidad. Al contrario, las máquinas no triviales dependen del estado interno de la máquina, por lo que no es posible asegurarse la obtención de un resultado a partir de la introducción lineal de un estímulo. Está claro que los seres humanos no son máquinas triviales, sin embargo, la comunicación educativa debe partir de la suposición de la trivialidad de los alumnos. Las causas de este fenómeno son variadas. En primer lugar, en el caso de que el sistema educativo no partiese de este hecho, la posibilidad de aprendizaje sería inexistente. En efecto, ¿cómo podría orientarse una comunicación destinada a la consecución de metas ante la imposibilidad de asegurar su efectividad? Como señala Robles, de esta paradoja resulta que la educación en sentido 
estricto es un imposible (2010). Por otra parte, el asumir que cada niño es un mundo en sí mismo (máquina no trivial), más allá de los deseos de la pedagogía, no haría posible el comenzar de un punto de partida común entre los alumnos, con lo cual la evaluación indiferenciada perdería sentido.

Esto no niega que sea posible elevar la complejidad de la trivialización, por ejemplo, en la dirección que señala Heinz von Foerster mediante las preguntas legítimas o preguntas cuya respuesta todavía no es conocida ni por el profesor ni por sus alumnos. Como se pregunta el científico austriaco, would it not be fascinating to think of an educational system that detrivilizes its students by teaching them to ask 'legitimate' questions, that is, questions for which the answer are unknow? (Robles, 2010). Sin embargo, incluso en este caso las premisas de trivialización no son desplazadas, sino que únicamente elevan su complejidad. Consecuencia de la aplicación del código, en la formulación de preguntas legítimas en el aula siempre se trata de respuestas correctas o incorrectas. Algunos alumnos aciertan y otros no lo hacen. Por ello, las individualidades son consideradas únicamente en tanto renuncien a estorbar la implementación del programa. La libertad es definida como aceptación de lo necesario, de hacer por la propia voluntad del alumno lo que los programas definen como requisito en la aplicación del código de selección social (Luhmann y Schorr, 1990: 57).

De esta forma, mediante evaluaciones, elogios y promociones a nuevos cursos el sistema de la educación formula sus propias diferencias. Si bien estas evaluaciones pueden tener un correlato en otros sistemas funcionales como el científico o el económico, adquieren su diferencia propia en el interior del sistema. Como señala Luhmann, la escuela no maneja la diferencia entre seno y no seno, sino entre los alumnos que saben manejar esta diferencia y los que no (Luhmann y Schorr, 1996: 46). En el mismo sentido, si bien la carrera puede pretender ser una satisfacción que el sistema educativo brinda al económico, su estructuración obedece a criterios internos al sistema. Producto de la diferenciación funcional, la aplicación del código de selección mejor/peor en la educación produce únicamente diferencias relevantes para el sistema educativo. La indiferencia frente a las comunicaciones de otros sistemas tiene como consecuencia que el "mejor" alumno no tenga asegurada por ser de tal forma una posición privilegiada en otros sistemas funcionales de la sociedad. La inclusión es un fenómeno contingente frente a la forma acumulativa de la exclusión (Luhmann, 1998: 176).

Este fenómeno se hace particularmente visible cuando analizamos el objeto de la educación, el niño. Esta es una abstracción creada por y para asegurar la continuidad de las operaciones del sistema educativo. En el mismo sentido que señalase Foucault respecto a la creación del hombre en el siglo XVII, el niño como medio de la educación posee una fecha de inicio $y$, probablemente, de término ${ }^{2}$. Como tal, no se refiere a ningún niño en específico -ni siquiera a uno deseado- sino a la generalización de un tipo de persona que, tras su paso por la escuela, es diferente de lo que hubiese sido de no haber pasado por ella (Luhmann y Schorr, 1996: 148). El hecho de si esto es o no posible de asegurar en máquinas no triviales es una cuestión que no abordaremos acá. Más allá de

Con el auge del aprendizaje continuo la observación del niño como objeto de la educación parece perder validez pues, ¿cómo compatibilizar esta tesis con la creciente necesidad en la vida adulta de obtener más titulaciones y certificaciones? Inspirado por Lenzen, Luhmann propone la alternativa del medio currículo vitae. Para una crítica del concepto véase Fernando Robles (2010: 13-17) y también Lars Qvortrup (2005). 
esto, el medio niño cumple su función de asegurar que la intención de educar alojada en los profesores tiene posibilidades reales de alterar psíquicamente a los alumnos, al hacer posible encontrar un correlato semántico de una persona que es posible transformar a partir de la aplicación de las metas formuladas en los programas.

Como puede seguirse hasta acá, el planteamiento expuesto hace innecesario considerar a la educación como rectora de la sociedad. Esto nos permite abordar desde esta teorización la desigualdad no como un problema de falta de coordinación del sistema educativo respecto a otros sistemas, sino como un problema en sí mismo, una consecuencia ineludible de la diferenciación funcional. En efecto, la selección social que el sistema lleva a cabo por la diferencia entre código y programa promueve la indiferencia respecto a ámbitos ajenos al educativo. Dicho lo anterior, queda todavía una deuda pendiente, especialmente en países tan desiguales como el chileno: ¿cómo es posible describir la relación estricta que existe entre el sistema educativo y las desigualdades provenientes de otros sistemas funcionales?; ¿no es esta relación lineal un argumento fuerte contra lo que hemos expuesto sobre la constante homogeneización del comienzo?

\section{LA UTILIZACIÓN DE LA FORMA INCLUSIÓN/EXCLUSIÓN EN EL SISTEMA ESCOLAR}

Como ha destacado Massé (2007), parece ser poco productivo describir la exclusión según el entendimiento tradicional del término en la teoría de sistemas. El sistema educativo presenta dos problemas en este ámbito: por una parte, la tendencia desarrollada hacia la inclusión universal y, por otra, la convivencia de formas de inclusión más o menos rígidas. En nuestro país, en el año 2008, la matrícula en los niveles básico y medio en comparación con la población total tendía hacia la universalidad, alcanzando un 94,6 y $80,5 \%$ en los niveles básico y medio respectivamente (MINEDUC, 2008). Según lo anterior, parece prudente afirmar que la exclusión se ha relegado a los niveles superiores de enseñanza. Por lo demás, al comparar las diferentes organizaciones que median la inclusión educativa distribuidas entre escuelas municipales, particular subvencionadas y privadas, esta uniformidad tiende a desaparecer. Como muestran los resultados de las pruebas de medición SIMCE y PSU, no tiene sentido argumentar sobre la equivalencia de acceder a estudios en estas escuelas.

Quedarse en este concepto de inclusión sería sin embargo desconocer los aportes realizados en aras de una mejor descripción de las dinámicas educativas. Sin un ánimo de exhaustividad, quiero dar cuenta de una serie de aportes que abordan el problema de la desigualdad a partir de la relación entre sociedad y educación, sin sacrificar la diferenciación del sistema educativo. En primer lugar se encuentra el aporte teórico y metodológico de Ossandon (2006). A partir de la definición de las bajas expectativas con las cuales los profesores de las escuelas municipales observan a sus alumnos, Ossandon propone complejizar la diferencia entre inclusión y exclusión mediante un paralelo con la conceptualización de Luhmann del riesgo, como la reentry entre controlable e incontrolable. De esta forma, la reintroducción de la diferencia en el lado de la inclusión da lugar a la distinción entre alumnos educables y no educables. Mientras de los primeros se esperan resultados educativos en el sentido tradicional del término, es decir, selecciones 
académicamente relevantes, sobre los segundos pesan objetivos mucho más orientados al disciplinamiento del cuerpo en línea con lo señalado por Foucault.

Sobre esta postura llama la atención, en primer lugar, la continuidad con los lineamientos de Luhmann sobre observar observadores y no estados del sistema (Luhmann, 1998: 33). En efecto, contrario a una parte importante de la investigación educativa basada en la descripción de propiedades supuestamente ontológicas de la realidad, Ossandon se interroga sobre los esquemas de diferencias que utilizan los observadores en la construcción de realidad. Producto de esta decisión, es ahora posible superar como categoría analítica la distinción entre público y privado -basada en el medio de propiedad de la economía- por una estrictamente educativa, en línea con la primacía descriptiva de la diferenciación funcional. Por último, la utilización de un enfoque orientado a observar las expectativas de los profesores sobre los alumnos tiene posibilidades de enlazarse con la prolífica literatura existente sobre la aplicación de la social selfufilling prophecy y el teorema de Thomas en la investigación educacional.

Una segunda propuesta teórica sobre el tema se encuentra en Robles (2005). Si bien no está específicamente abocada al tratamiento de la exclusión en la educación, su descripción de las categorías que median la individualización e individuación en la sociedad contemporánea puede ofrecer importantes beneficios para la investigación empírica. Producto de la combinación de las categorías clásicas de inclusión y exclusión, Robles obtiene una representación más precisa de los procesos de construcción de domicilios sociales: la inclusión entendida en el sentido tradicional (inclusión en la inclusión); inclusión, pero sin redes de soporte suficientemente conformadas para asegurar su permanencia (exclusión en la inclusión); exclusión con mecanismos de asociación y resistencia (inclusión en la exclusión) y exclusión entendida en el sentido tradicional del término (exclusión en la exclusión).

Las nuevas categorías de exclusión en la inclusión e inclusión en la exclusión permiten observar formas de exclusión que subyacen (¿sostienen?) la inclusión educativa, permitiendo dar cuenta de características asociadas a la exclusión, como la observación corporal carente de premisas comunicativas en contextos tradicionalmente vistos como integrados (Luhmann 1998: 194). Por otra parte, la categoría de inclusión en la exclusión hace posible la descripción de redes orientadas a la educación en contextos de exclusión, aspecto que la sociología de la educación, en tanto sociologías de las instituciones escolares no ha tenido debidamente en cuenta (Bonal 1998). En último lugar, ambas categorías permiten integrar teóricamente la fragilidad de la inclusión junto al carácter acumulativo de la exclusión en la educación.

La postura de Mascareño comprende un énfasis en la negligencia característica de los sistemas sociales en la diferenciación funcional $(2000,2005)$. Mediante un recorrido histórico por la diferenciación del sistema educativo, el sociólogo chileno describe la desigualdad no mediante la postura de la sociología crítica de definir la educación como un área manipulada por las relaciones económicas, sino más bien como una consecuencia ineludible de la diferenciación sistémica. Dado que las posiciones en el interior de escuelas y universidades son asignadas únicamente mediante la selección social, las diferencias sólo son atribuibles a esta (Luhmann y Schorr, 1996: 57). De esta forma, la propuesta de Mascareño entiende la desigualdad educativa como el producto de la permanencia de resabios de estratificación en la sociedad contemporánea que, dada la primacía funcional, debe acomodarse a esta forma de diferenciación. 
Por último, tenemos el aporte de Michailakis y Reich derivado de su investigación sobre las formas de exclusión que afectan a los limitados intelectuales en Suecia (2009). Partiendo de la definición que Luhmann desarrolla junto a Schorr de que en el sistema educativo se cumplen funciones tanto de educación como de selección, los autores asumen que el cumplimiento casi universal de la primera función no debe hacer omitir problemáticas asociadas a la segunda. Según este planteamiento, en el caso de cierto tipo de alumnos, la promoción a un nivel superior no sería consecuencia de la aprobación en los mecanismos de selección (evaluaciones), sino más bien un producto temporal ineludible. De forma paradójica, la exclusión educativa se encuentra asociada en esta propuesta a la no aplicación del código de selección.

La principal utilidad de esta postura es que establece la función de selección desde una observación carente de premisas morales, sin por ello sobrecargar la atribución de posiciones superiores e inferiores, como un predictor lineal de la inclusión en el resto de sistemas funcionales. Esta argumentación está en línea con la diferenciación entre código y programa (Luhmann y Schorr, 1990, 2000). En efecto, la generación de diferencias en forma autopoiética no puede llevarse a cabo sin selección social. Por otra parte, dado que el código sólo distingue comunicaciones educativas no puede asegurarse la inclusión en otros sistemas funcionales por estar incluido en el sistema educativo pues, a fin de cuentas, la inclusión no es acumulativa como sí lo es la exclusión. Por último, si bien la presente opción teórica posibilita observar la desigualdad como consecuencia de la no aplicación del código, esta postura permite también dar cuenta de los efectos sobre los niveles, tanto organizacionales como de interacción.

En esta sección he descrito cuatro autores que intentan observar la desigualdad en el sistema educativo como un producto de sus propias operaciones sin concebirlo como el centro articulador de lo social, como lo hacían las teorías funcionalistas y las sociologías de influencia marxista y del capital humano. Quiero ahora sintetizar las posibilidades de estos lineamientos en la siguiente Tabla a partir de su comparación respecto a los supuestos de la teoría de sistemas educacionales y las posibilidades de relación con otras teorías no sistémicas.

Tabla 2. Comparación entre supuestos de la teoría de sistemas educacionales y otras teorías no sistémicas

\begin{tabular}{|c|c|c|c|c|}
\hline & Ossandon & Robles & Mascareño & $\begin{array}{c}\text { Michailakis } \\
\text { y Reich }\end{array}$ \\
\hline $\begin{array}{c}\text { Teoría de la } \\
\text { diferenciación }\end{array}$ & + & + & + & + \\
\hline $\begin{array}{c}\text { Selectividad } \\
\text { pedagógica }\end{array}$ & \pm & \pm & + & + \\
\hline Medio del niño & + & + & \pm & \pm \\
\hline $\begin{array}{c}\text { Homogeneización } \\
\text { del comienzo }\end{array}$ & + & \pm & + & + \\
\hline $\begin{array}{c}\text { Relaciones fuera } \\
\text { de TSS }\end{array}$ & \pm & - & \pm & - \\
\hline
\end{tabular}


El abandono de las observaciones que asocian a la educación con el ámbito central de la sociedad está mediado por la utilización de la teoría de la diferenciación funcional. Sobre este punto existe un consenso en estas posturas sobre que la escuela no es la instancia socializadora que permite el orden social, sino que asigna posiciones a partir de operaciones internas. De esta forma, los aspectos económicos o políticos juegan un rol secundario únicamente integrable a la codificación sistémica mediante los programas. En diferentes niveles de análisis todos los autores apuntan a esta característica. Mientras Ossandon otorga un mayor valor a las expectativas sobre el educando, Mascareño apunta a la descripción de las formas que toman los resabios de estratificación en un sistema educativo diferenciado. Nótese que ambas posturas no son opuestas: es perfectamente admisible que elementos de la sociedad estratificada tomen formas propias de la diferenciación funcional mediante la distinción entre alumnos educables y no educables.

En lo referente a la selectividad pedagógica, existe una mayor matización entre los autores señalados. Si bien todos presuponen la generación de diferencias autopoiéticamente, en algunos esto se analiza con detalle, en tanto otros únicamente dejan implícitas sus posibilidades. En este sentido, Ossandon y Robles dejan pendiente la profundización acerca de la forma en que influyen las expectativas docentes y la exclusión en la inclusión en la selectividad pedagógica. Si, como argumenta Robles, se está en presencia de un sistema educativo dual como consecuencia de la interferencia del medio dinero en las operaciones sistémicas, ¿en qué medida esto queda de manifiesto en la utilización de una selección menos pedagógica, es decir, menos centrada en la evaluación de premisas académicas? Idéntica interrogante es posible realizar en el trabajo de Ossandon, pese a que sus consecuencias permiten aventurar la primacía de una selección disciplinaria antes que pedagógica sobre los alumnos observados como no educables. En el caso tanto de Mascareño como de Michailakis y Reich, la imposición del código de selección cumple un rol importante como el espacio donde la exclusión educativa se realiza en la sociedad funcionalmente diferenciada.

El niño como medio de la educación es abordado en diferentes niveles entre los autores. Ossandon profundiza en la importancia de la interacción entre profesor y alumno, particularmente la distinción utilizada sobre el educando. En el caso de Robles, si bien en el estudio de las categorías de inclusión y exclusión no aborda la construcción del niño, sí lo hace en otro lugar, donde analiza la evolución histórica desde la violencia física como medio de irritación simbiótico en el siglo XIX hasta la primacía en la actualidad de la apelación valórica (2010). Por su parte, Mascareño no concentra sus esfuerzos en el niño, sino en la subordinación de la educación a las directrices de la diferenciación funcional y la transformación histórica en sus funciones orientadoras (2000). En el mismo sentido, Michailakis y Reich tampoco abordan el tema específico, si bien su concepción de la exclusión como ausencia de selección se basa en el supuesto de un niño con menores posibilidades de aprendizaje que es excluido tanto en los niveles de interacción como de organización.

En relación a la homogeneización del comienzo, este presupuesto de la educación es asumido pero no explicitado en la mayoría de los estudios. Este es el caso de Ossandon, que, si bien no incorpora una reflexión en este punto, hace posible entrever una preocupación sobre el tema a partir de las expectativas asociadas. En efecto, en la articulación de las posiciones educativas autopoiéticamente se encuentra el presupuesto de un comienzo homogeneizado para producir heterogeneidad documentada (Luhmann y Schorr, 1996: 
51). Esto deja abierta la cuestión, sin embargo, de la reiniciación del comienzo pues, si los profesores diferencian inicialmente entre dos tipos de alumnos, ¿cómo puede argumentarse sobre un comienzo indiferenciado de los alumnos? En el estudio de Robles tampoco existe esta preocupación. Pese a ello, es posible enlazar desde otro estudio su consideración sobre un sistema dual de educación intervenido por medios económicos (2010) con la diferencia entre no educable y educable propuesta por Ossandon. Mascareño, por su parte, determina la homogeneización del comienzo como parte fundamental de la selectividad pedagógica. Por último, en Michailakis y Reich este problema es abordado sólo parcialmente. La exclusión de la función de la selección implica la ausencia de evaluaciones, fenómeno por el cual la necesidad de remitir a un nuevo comienzo deja de ser un problema en el aula.

Sobre las posibilidades de relación fuera de la teoría de sistemas, la investigación de Ossandon parece ser relacionable con los estudios sobre expectativas en el ámbito docente. La literatura sobre esto es extensa y proviene desde el estudio de Rosenthal y Jacobson sobre el efecto Pigmalión (1968). Paradójicamente, las categorías propuestas por Robles en torno a la inclusión y la exclusión pueden verse complementadas con estudios derivados de la teoría del capital humano. En un estudio realizado posteriormente (2010) el autor fundamenta su descripción de un sistema dual de educación a partir de estadísticas relativa al quintil de ingreso familiar. Por su parte, Mascareño, en un estudio sobre las expectativas puestas en la educación, introduce la posibilidad de combinar la revisión historiográfica con este objetivo (2000). En último lugar, la posición de Michailakis y Reich parece tener menores posibilidades de asociación con ideas fuera de la teoría de sistemas. Esto puede deberse a que la reflexión sociológica sobre el código de selección no se ha desarrollado tan ampliamente como la llevada a cabo sobre la comunicación educativa (Luhmann y Schorr, 1990: 58).

\section{REFLEXIONES FINALES}

Las alternativas mencionadas están lejos de ser las únicas provenientes desde la teoría de sistemas. No pretender ser una solución definitiva a los problemas tradicionales de la sociología de la educación, sino más bien ofrecer una alternativa a las descripciones que parten de la premisa de la educación como centro de lo social. A mi juicio, esta selección hace posible observar características específicas de los sistemas educativo sin subordinar sus operaciones a lógicas de otros sistemas sociales. En este sentido, hacemos nuestra la crítica de Bernstein a estos tipos de sociología. "Muchos de los análisis (...) asumen el auténtico discurso que someten a su análisis. En particular, estas teorías consideran el discurso pedagógico como medio para otras voces: clase social, género, raza. Los discursos de educación se analizan por su poder para reproducir relacionantes dominante/ dominado, externas al discurso que, sin embargo, penetran en las relaciones sociales, los medios de transmisión y la evaluación del discurso pedagógico" (Bernstein, 1993).

Reconozco que la posición expuesta, derivada de la teoría de sistemas, tiene varios aspectos problemáticos. La trivialización del alumnado y su subordinación al funcionamiento de los programas puede resultar chocante para una pedagogía centrada en la liberación (destrivialización) del alumno mediante el conocimiento. La imposición de criterios evaluativos orientados a seleccionar entre mejores y peores alumnos parece 
también resultar ajena a los supuestos fines de igualdad de la educación. Como señala Luhmann, estamos demasiado acostumbrados a confundir la reivindicación de los movimientos sociales sobre igualdad de oportunidades con la eliminación del carácter selectivo de la educación (Luhmann y Schorr, 1990). Si bien la socialización puede ser una alternativa a la educación, pues permite superar la resistencia del alumnado, falla al no estar orientada a la consecución de metas específicas y no poder evaluar los logros sistemáticamente. En idéntico sentido, la imposibilidad de derivar efectos lineales de las posiciones asignadas en la sala de clases parece atacar el sentido común desde el cual la sociología de la educación se ha construido como apuntamos en la primera parte de este trabajo. Pese a lo anterior, estas contradicciones no son suficientes para aceptar o rechazar a priori las descripciones expuestas.

Con esto damos por finalizada nuestra propuesta de una alternativa a la observación de la educación como el centro de la sociedad moderna. Sería un error asumir que esta es una perspectiva decantada de una vez y para siempre. En particular, el presente trabajo ha intentado mostrar la presencia de un discurso común en torno a la centralidad de la educación para coordinar el orden social en las teorías funcionalistas, críticas y del capital humano. La opción de la teoría de sistemas parece favorecer en este plano la observación de la educación como un orden emergente autónomo, lo cual hace posible dar cuenta de sus características específicas. Por último, hicimos una pequeña revisión de algunas propuestas teóricas que se han realizado desde la teoría de sistemas para abordar la desigualdad en la educación junto a sus posibles conexiones con investigaciones no sistémicas.

Bajo la utilización de nuevos esquemas de diferencias, la sociología de la educación tiene la oportunidad de dejar de ser observación de la unidad social representada por la educación para decantar en observación de la diferencia, de lo que distingue al sistema funcionalmente diferenciado de la educación respecto a los demás sistemas. Si bien es posible que la educación haya sido central en los tiempos que los clásicos de la disciplina tuvieron que enfrentar, sería un error continuar utilizando su acervo intelectual en una sociedad radicalmente diferente. De haber motivado este trabajo a la reflexión en esta dirección el objetivo está más que logrado.

\section{REFERENCIAS BIBLIOGRÁFICAS}

Althusser, L. (1985). El aparato ideológico del estado escolar como aparato dominante. En Gras, A. (comp.), Sociología de la educación, Textos fundamentales. Madrid: Narcea.

Bernstein, B. (1990). La estructura del discurso pedagógico. Madrid: Morata.

Beyer, H. s/f. La urgencia de la educación. Santiago: Centro de Estudios Públicos.

Bonal, X. (1998). Sociología de la educación. Una aproximación crítica a las corrientes contemporáneas. Madrid: Editorial Paidós.

Bernstein, B. (1985). Clasificación y enmarcación del conocimiento educativo. Revista Colombiana de Educación, vol. 15.

Bourdieu, P. y Passeron, J. (1964). Los herederos. Los estudiantes y la cultura. Buenos Aires: Siglo XXI.

Bourdieu, P. y Passeron, J. (1977). La reproducción. Elementos para una teoría del sistema de enseñanza. Barcelona: Laia.

Bowles, S. y Gintis, G. (1976). Schooling in Capitalist America. Nueva York: Basic Books. 
Coleman, J. (1966). Equality of educational theory. Washington DC: U.S. Office of Education.

Durkheim, E. (1991). Educación y sociología. México: Editorial Colofón.

Jencks, C. (1973). Inequality in retrospect. Harvard Educational Revien, vol. 43.

Luhmann, N. (1994). Unidad y diferenciación en la sociedad moderna. Ponencia presentada en el Congreso Internacional de Sociología en Bielefeld, Alemania. Julio de 1994.

Luhmann, N. y Schorr, K. (1996). Teoría de la sociedad y pedagogía. España: Ediciones Paidós Ibérica.

Luhmann, N. (1998). Complejidad y modernidad. De la unidad a la diferencia. Madrid: Editorial Trotta.

Luhmann, N. y Schorr, K. (1990). Presupuestos estructurales de una pedagogía reformista. Análisis sociológicos de la pedagogía moderna. Revista de Educación, $n$. 291, 55-79.

Luhmann, N. y Schorr, K. (2000). Problems of reflection in the system of education. New York: Waxmann.

Mascareño, A. (2000). La ironía de la educación en América Latina. Revista Nueva Sociedad, $n$. $165,109-120$.

Mascareño, A. (2005). La imposibilidad de la igualdad por la vía educativa. Informe $\mathrm{N}^{\circ} 513$ Educación. Valparaíso: Universidad Católica de Valparaíso.

Mascareño, A. (2005). Sociología de la felicidad: lo incomunicable. Teología y Vida, vol. XLVII, 190-208.

Mascareño, A. (2006). Sociología de la solidaridad. La diferenciación de un sistema global de cooperación, En Arnold, M. y Thumala, D., ed. Colaboración, cultura y desarrollo. Santiago: Ediciones Universidad de Chile.

Mascareño, A. (2009). Medios simbólicamente generalizados y el problema de la emergencia. Cinta Moebio, n. 36, 147-197.

Massé, C. (2007). El sistema de la educación en Luhmann desde una perspectiva crítica. Cinta de Moebio, n. 30.

Michailakis, D. y Reich, W. (2009). Dilemmas of inclusive education. European Journal of Disability Research.

MINEDUC (2008). Chile hacia el 2050-Proyecciones de Población 1990-2020. Santiago: Departamento de Estudios y Desarrollo, Instituto Nacional de Estadísticas.

Ossandon, J. (2002). Objeto pedagógico perdido. Una interpretación 'Luhmanniana' de las bajas expectativas en liceos subvencionados en Chile. Santiago: Universidad de Chile, Chile.

Parsons, T. (1976). La clase escolar como sistema social: algunas de sus funciones en la sociedad americana. Revista de educación, n. 242, 64-86

Qvortrup, L. (2005). Society's Educational System. An introduction to Niklas Luhmann's pedagogical theory. Seminar.net, volume 1, $n$. 1. Disponible en: http://www.seminar.net/files/LarsQvortrupSocietysEdSystem.pdf

Robles, F. (2005). Contramodernidad y desigualdad social: Individualización e individuación, inclusión/exclusión y construcción de identidad. La necesidad de una sociología de la exclusión. Concepción: Universidad de Concepción.

Robles, F. (2010). Las paradojas de la educación chilena y la apología de las máquinas triviales. ¿Ha llegado la hora del cambio en el sistema educacional? Disponible en http://es.scribd.com/ doc/76090964/EL-CAMBIO-EDUCACIONAL.

Rosenthal, R. y Jacobson, L. (1968). Pygmalion in the classroom: teacher expectation and pupils intellectual development. New York: Holt, Rinehart y Winston.

Schultz, Th. W. (1983). La inversión en capital humano. Educación y Sociedad, vol. 1, 180-195. 